\title{
Use of Human-Centric Functional Modeling to Maximize Convergence in Integrative Research
}

\author{
Andy E. Williams, Nobeah Foundation, Nairobi, Kenya
}

\begin{abstract}
This paper leverages a specific approach to conceptual modeling (Human-Centric Functional Modeling or HCFM) to suggest an objectively measureable definition of convergence in research, and outlines an argument for the conclusion that the use of HCFM in representing any static conceptual entity such as a reasoning process, or any dynamic conceptual entity (capable of changing states) such as a biological or physical system, is required in order to remove specific limits to the capacity of that research for convergence in human understanding between disciplines. And therefore the use of HCFM is required in any discipline of study, whether physics, mathematics, economics, religion, philosophy, or other, in order to maximize the capacity for convergence in that research within the capacity of individuals or groups to conceive concepts in those fields. Specific examples demonstrating the use of HCFM to achieve unprecedented convergence in research are presented. And the logical argument that HCFM is required to maximize convergence within current individual or group limits is summarized as a theorem as the first step in defining a mathematical proof.
\end{abstract}

\section{Keywords}

Human Centric Functional Modeling, Functional Modeling Framework, General Collective Intelligence, Convergence

\section{Introduction}

The approach to conceptual modeling presented in this paper is believed to be an important innovation that contributes a significant advance to approaches in the existing conceptual modeling literature [1] - [6] in that it leverages what is believed to be the first complete functional model of cognition to gain the capacity to base conscious conceptual modeling on the same representation of concepts that is proposed to be used natively and unconsciously in the brain. This approach creates the capacity to see greater commonality between a wider range of ideas so that patterns of solutions can be reapplied, with the result that some long-standing problems such as consciousness or cognition that have so far proved intractable now have promising potential solutions. For this reason this conceptual modeling approach is a potentially disruptive innovation with regards to convergence research, which seeks commonality between understanding developed in different disciplines. The claim of this paper is that this approach, called Human-Centric Functional Modeling (HCFM), has the capacity to introduce unprecedented convergence in all subjects within the individual or group's capacity to conceive, while also facilitating collective cognition to create the potential to significantly increase that collective capacity. Before exploring the evidence for these claims, and why should this be the case, definition of HCFM, and definition of the models of individual and group cognition and cognitive capacity used to support this claim, are necessary.

\section{Human-Centric Functional Modeling}

In this approach, conceptual entities (any entity that can be conceived) are separated into two categories; static entities that don't change state, and dynamic entities that do. Static entities are described in terms of their functions, identified by the outcomes the concepts can be observed as functioning to achieve. Dynamic entities are also described in terms of function, but instead the minimal set of observed functions by which they change state, and from which all other observed functions can be composed. This process of observation is human-centric because it is limited to observations that can be made within innate human perception rather than being in terms of constructs such as beliefs or intellectual theories about the mechanisms by which those functions are implemented, or in any other terms that can't yet be validated within observation. 
Dynamic entities (systems) are not only described in terms of the minimal set of human-observable functions capable of describing all their behaviors, but in addition are described in terms of the states accessible through these functions. The set of these states forms a "functional state space" through which the system moves. In addition, the system being operational (alive in the case of biological organisms) is represented as the system being confined to a bounded region of a "fitness space" that represents its fitness to execute all its functions. By defining boundary conditions that the system must adhere to in order for its dynamics in this fitness space to be sustainably stable, it is possible to identify equations that meet those boundary conditions. And by defining the impact on fitness of any change in functional state, it is possible to define algorithms by which the system can navigate its state space in order to satisfy those equations. In this way, HCFM can be used to develop models that describe the entire set of behaviors of complex systems, even where the mechanisms by which those behaviors are implemented are unknown.

A Functional Modeling Framework (FMF) [7] developed using this approach has been used to define models for all living systems as a hierarchy of adaptive problem-solving processes through which each organism adapts to solve problems posed by its internal and external environment. Functional modeling has often been used to facilitate the engineering of complex system by multidisciplinary teams by identifying functional components with well-defined interfaces that remove the need for one discipline to understand another. This in turn removes the barriers to scaling multidisciplinary cooperation in the design and development of complex systems. In the case of cognition, defining a set of functions with which all cognitive models can be compared has the potential to remove the barriers to scaling research cooperation, enabling massively collaborative research efforts to reliably converge on a single understanding of cognition that maximizes fitness in representing observed functionality, where difficulty of communication between different disciplines studying the problem has previously made such massive collaboration impossible in practice.

Models implementing this hierarchy of problem-solving processes include a model of consciousness [8], [9] with the potential capacity for self-awareness, a model of individual and artificial cognition with the potential capacity for human-like general problem solving ability [10], as well as models for more basic processes like homeostasis. Furthermore, because the model of cognition is a functional model independent of implementation, it has been used to define a model for collective cognition or General Collective Intelligence (GCI) with the potential to give groups vastly greater ability to solve collective problems [11], including any research [12].

Because the set of functions used to represent human cognition must by definition be Turing complete if they are correct, and because they are potentially reversible, this set of functions has been suggested as the basis for logic gates in a quantum computer, to significantly expand the uses where quantum computing might be applied, as well as expanding the environmental conditions (e.g. temperature) at which it might be applied. In the case of AI-driven innovation, this functional modeling approach creates the potential to combine all AI models and all training data into a single library that an AGI might use to increase its general problem solving ability[12]. However, its application to quantum computing is still highly speculative since this hasn't been explored in detail.

But there is significant motivation to change that. This approach reveals current AI systems as solving the wrong problems for maximizing impact on problems related to collective well-being. The framework predicts that lack of the complete hierarchy of adaptive problem-solving domains in current AI must result in an imbalance between centralization of processes where optimal, and decentralization of processes where optimal, resulting in an alignment with the interests of centralized decision-makers 
even where not advantageous in maximizing collective outcomes. This misalignment is a hidden bug that isn't visible without this functional modeling approach. In part due to this misalignment, the framework predicts that entire classes of group problems that might be existential threats to human civilization cannot reliably be addressed without GCI [13]. It also suggests that AGI must tend to drive dangerously destabilizing levels of inequality without GCI to create chains of cooperation complex enough to bind AGI to collective human well-being. Combined with the fact that proving the FMF based model of GCI may also prove the FMF based model for AGI, this suggests that GCI is potentially a far more transformational and disruptive innovation than any standalone approach to AGI itself [14].

In developing functional models for consciousness, cognition, and every other biological process, HCFM and the FMF it was used to develop have been demonstrated to have the capacity to be used to develop convergent theories of dynamic systems in a wide variety of areas. In addition, HCFM is also currently being applied to philosophy, religion, economics, and other topics in which static conceptual entities such as reasoning must be compared.

\section{Limits to Cognitive Ability}

In the FMF all concepts accessible to a system of cognition are represented as forming a "conceptual space". This conceptual space is the functional state space of the cognitive system. The cognitive system navigates between concepts using reasoning processes. General problem solving ability in the FMF is the ability to sustainably navigate the conceptual space in a way that can potentially span the entire space and therefore navigate from any problem to any solution. The level of general problem solving ability (intelligence) is the volume of this space that can be navigated per unit time.
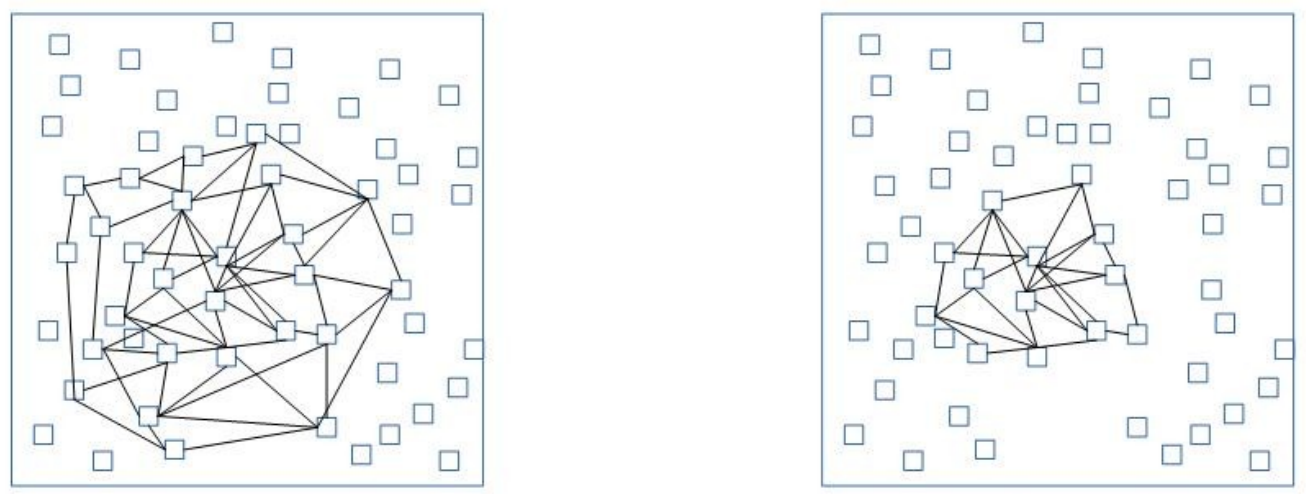

Figure 1: General problem-solving ability: a larger volume of conceptual space navigated per unit time (left) represents higher general problem solving ability. A smaller volume of conceptual space navigated per unit time (right) represents lower general problem solving ability.

Any category of problem definition that can't be conceived in a given topic is outside of that entity's capacity to understand (outside of the limits of it's cognitive ability). 

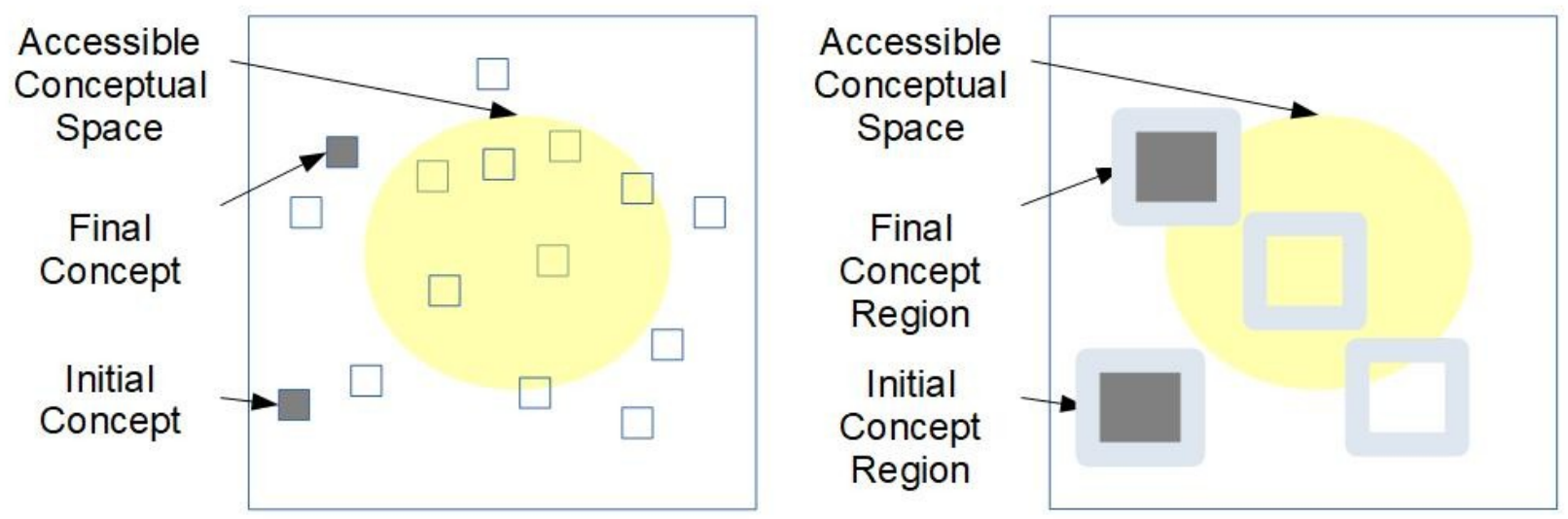

Figure 2: Undefinable problems include those (left) for which initial and/or final concepts in the problem definition are outside accessible conceptual space, or (right) initial, or final concepts in problem definition are not sufficiently resolvable to be found within the accessible region.

A solution or reasoning process is a path through conceptual space. Any category of solution that can't be conceived in a given topic is also outside of that entity's capacity to conceive (again outside of the limits of their cognitive ability).
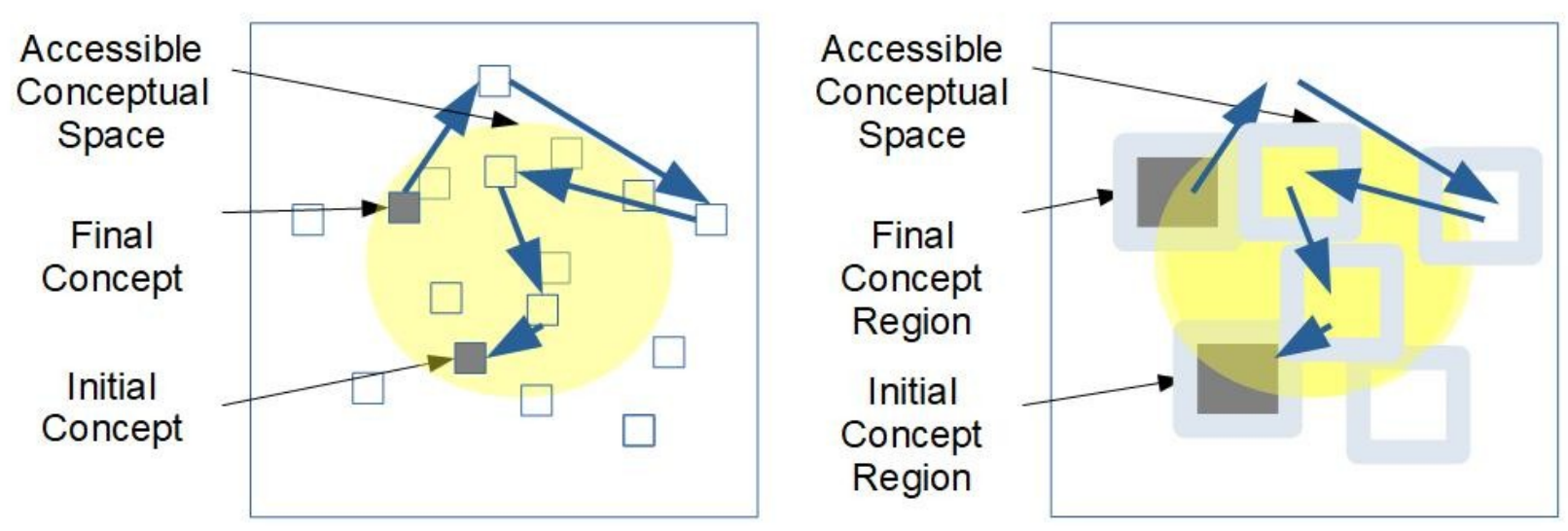

Figure 3: Undiscoverable solutions include those (left) in which the reasoning required exits accessible conceptual space, or those (right) in which the initial, final, or intermediate concepts in the reasoning are not sufficiently resolvable to be found within the region.

In group or collective cognition, the model of GCI suggests that the intelligence of the group is an emergent property of the system by which group reasoning is orchestrated. This intelligence is distinguished from the intelligence of any individual group member in that the collective intelligence is intrinsically aligned with collective well-being (measured in fitness to achieve all functions), where the intelligence of the individual is intrinsically aligned with individual well-being. Without a definition of collective well-being and without an adaptive problem solving system to maintain stability of fitness in that collective well-being, this model suggests that very little collective reasoning is achievable. Once a system with the capacity for GCI has been introduced, the same limits to cognition above, namely the limits to problems or solutions that can be perceived or conceived, are also believed to apply. These limits to the generation of ideas themselves will of course define the limits to convergence of ideas. 
Human thought has been defined as being comprised of intuitive thought, and rational methodical thought [17]. The FMF models both intuitive and rational methodical thought processes in terms of common functional models having input concepts, output concepts, and a set of additional concepts that determines context of execution. Rational methodical processes are represented as being like "equations". In order to be used, an equation must exist, the values of the input variables must be known, and the cognitive system must have the capacity to execute it. As a result rational connections between concepts are quite restrictive. In the FMF intuitive processes are represented as "black boxes"

that detect patterns of past solutions. Intuitive connections are far less restrictive since they only require sharing any one of a very large set of potential categories between concepts. For these reasons, intuitive relationships might be far less accurate in being navigated consecutively, and therefore less useful in a series of very precise steps like engineering a bridge. Rational methodical processes on the other hand might be far less accurate in being navigated massively in parallel, and therefore less useful in simultaneously considering a vast set of information and quickly coming up with an answer, like a musician improvising in a jazz band. Neither process is better than the other. But the importance of these considerations is that using each in it's appropriate context might significantly impact cognitive ability. And as mentioned, cognitive ability might set a limit to the possibility of convergence.

Two regions in conceptual space defined according to HCFM might not be logically connected in the sense of not having repeatable, rational methodical reasoning processes connecting them. However, any two regions can always be connected by intuitive reasoning, which requires only the ability to assign a common category to concepts. In the case of the reasoning processes representing any function that defines any static or dynamic concept, this category might apply to any of the properties of its functional model, including inputs, outputs, the outcomes associated with those outputs, or the context of execution. Such relationships based on categorization according to "black boxes" are by definition subjectively defined. On the other hand, any minimal set of functions defined in HCFM to span a conceptual domain must be rational methodical reasoning processes if they are to span that space in terms of objectively well-defined concepts. In this sense, increasing cognitive ability, and therefore increasing ability to perceive and conceive concepts that can be converged, requires increasing capacity for rational methodical reasoning.

Finally, another limit to cognitive ability, and therefore convergence, is also memory, which defines the size of the conceptual space.

\section{Convergence}

Intuitively, convergence occurs when the same concept can be used to describe two different phenomena. In the FMF, a concept is defined by its relationships to other concepts. All such relationships are represented as reasoning processes, which in turn are represented as paths from one concept to another in the conceptual space.

This paper proposes to define convergence in understanding as the overlap in the set of reasoning processes $[\mathrm{P}]_{1}$ required to describe concept $\mathrm{C}_{1}$, and the set of reasoning processes $[\mathrm{P}]_{2}$ required to describe $\mathrm{C}_{2}$. This definition can be validated as being correct at two sets of boundary conditions, one in which there is complete convergence in that category, and the other in which $\mathrm{C}_{1}$ and $\mathrm{C}_{2}$ belong to mutually exclusive categories, in which case there is zero convergence in those categories 

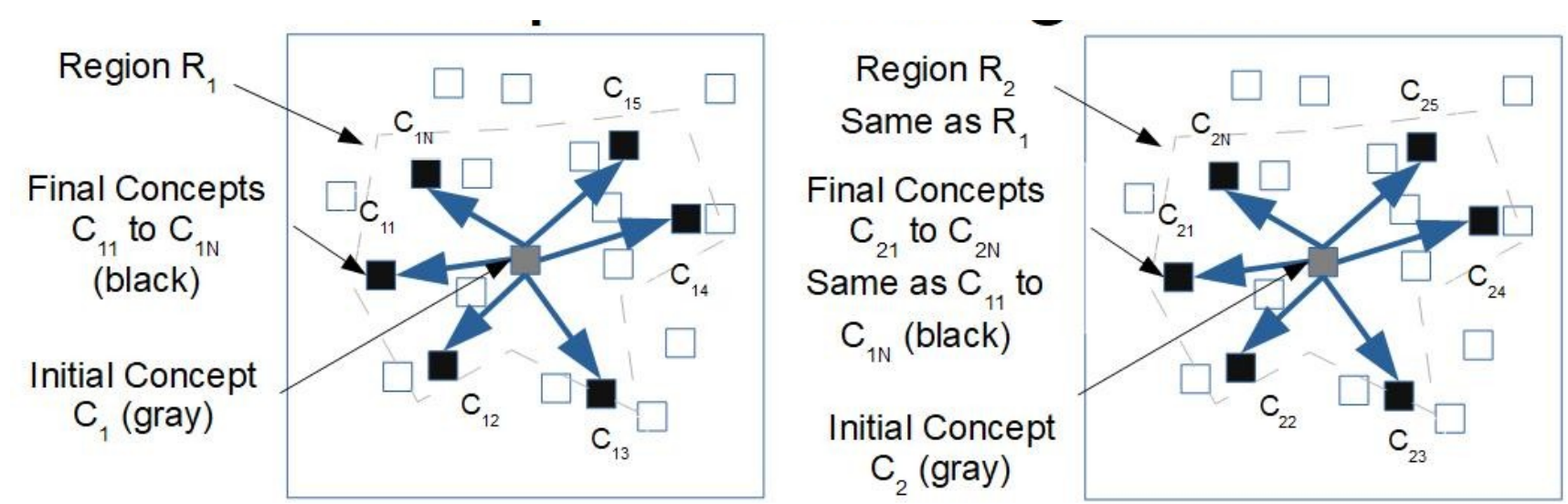

Figure 4: Complete convergence occurs when one concept (left) falls in the same category as another (right), so the regions of conceptual space required to describe the two concepts is the same. In partial convergence some concepts in a category apply, in which case the regions might be expected to overlap.

In the first case, complete convergence occurs when two concepts $\mathrm{C}_{1}$ and $\mathrm{C}_{2}$ belong to the same category, in which there is complete convergence of the two concepts within that category. In the second case, the concepts $\mathrm{C}_{1}$ and $\mathrm{C}_{2}$ belong to mutually exclusive categories, in which there is zero convergence between the two concepts within those categories.
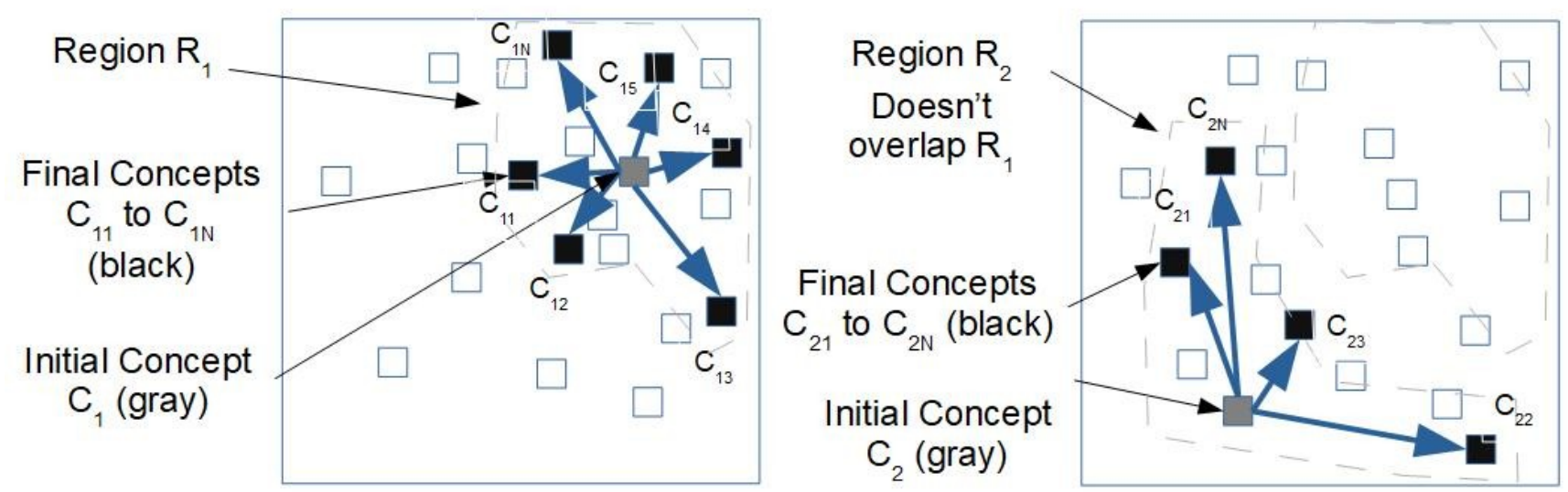

Figure 5: Complete lack of convergence (non-convergence) occurs when the region of conceptual space required to describe one concept (left) does not overlap with that required to describe another (right).

In the specific case of defining a set of cognitive functions to represent the entire cognitive space (i.e. to represent all the functional states of the cognitive system) then in order to be considered "minimal", any minimal set of functions must be capable of representing any other minimal set of functions.

Furthermore since any minimal set of functions must have the capacity to represent all the cognitive system can do, it must have the capacity to represent all valid functions of any model of cognition. Therefore the minimal set of functions required to represent any one model of cognition must be the same as the minimal set for any other model. Because any minimal set of reasoning processes implies maximum generalizability, and because generalizability is a measure of convergence, then any minimal set of functions required to unambiguously specify a concept also represents maximum convergence. Since the minimal set must also be navigable within human cognition (for example representing 
concepts in binary form might represent a very simple set of operations but is not human-navigable), then maximum convergence must occur with the use of a HCFM to obtain a minimal set of functions.

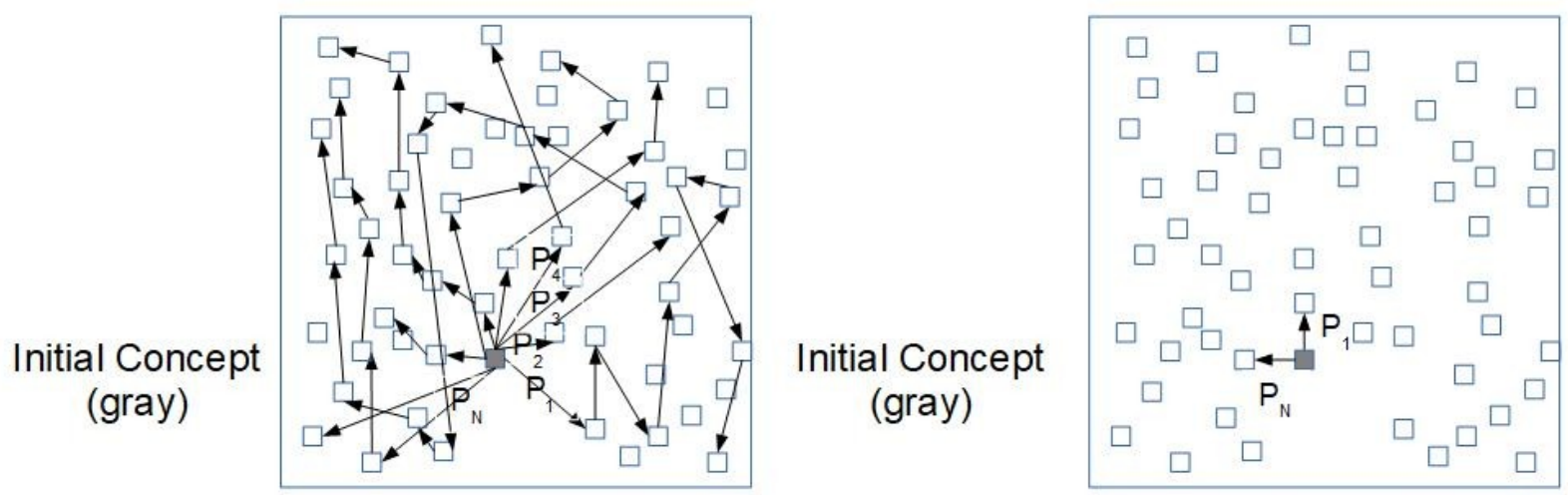

Figure 6: The relationship between convergence and minimal reducibility is that a set of reasoning processes $[P]_{N}$ might span the conceptual space. However, the more complex those processes (left), the less reusable they are in navigating to other positions in conceptual space, and the greater the memory and other cognitive resources required to store, retrieve, and execute each one. Therefore the greater the difficulty in navigating the conceptual space. A minimally reducible set of reasoning processes $[P]_{N}$ that spans the conceptual space (right) can be maximally reused.

\section{Theorem}

Assume that a given region of conceptual space $\mathrm{R}_{1}$ is required to unambiguously describe a concept $\mathrm{C}_{1}$, and another region $R_{2}$ is required to describe concept $C_{2}$. $R_{1}$ consists of all the concepts around $C_{1}$ and the reasoning processes $[\mathrm{P}]_{\mathrm{N}}$ required to navigate between them. If convergence in understanding is defined as the overlap in the set of reasoning processes $[\mathrm{P}]_{1}$ required to describe concept $\mathrm{C}_{1}$, and the set of reasoning processes $[\mathrm{P}]_{2}$ required to describe $\mathrm{C}_{2}$, and $[\mathrm{M}]_{1}$ is the minimal set of reasoning processes capable of spanning all $\mathrm{R}_{1}$, and $[\mathrm{M}]_{2}$ is the minimal set of reasoning processes capable of spanning all $\mathrm{R}_{2}$, then maximum convergence occurs when the minimal set of reasoning processes (functions) is chosen. Let the set of reasoning processes that are truthful [15] within human processes of observation be defined as $R_{C}$. Then $[M]_{1}$ is equal to $[M]_{2}$ and both are within $R_{C}$.

This theorem remains to be proven. If true, this theorem suggests that HCFM is required to remove the limits to maximizing convergence in any research and any other processes (to the capacity of the individual or group cognition to perceive or conceive ideas that can be converged).

\section{Practical Examples of Convergence}

In the case of divisive subjects such religion or politics, conflicting beliefs cannot fit into the same conceptual space as defined in the FMF unless one is categorized as untrue. In groups this segmented representation of knowledge prevents the addition of all knowledge into a single collective conceptual space that a GCI can use make a group more intelligent in navigating. In HCFM entities are no longer defined by any "truths" other than truths in the category of their function. In other words it is no longer important whether a given belief is a historical truth. What is important is what the belief in that concept functions to accomplish. From this point of view, faith and the thankfulness accompanying it might be seen as having the powerful function of promoting gratefulness, and gratefulness might have some function, perhaps maximizing the degree to which one believes one has everything one needs, which in turn could maximize the degree to which one seeks usefulness in what one already has, rather than squandering it. 
From the perspective of HCFM, all truths other than outcomes that a concept functions to achieve become subjective opinions. As shown in the segment of conceptual space in figure 7, conflicting subjective facts can be accommodated in the same conceptual space (i.e. it doesn't matter if subjective facts are true). Since functions can be shared by different concepts, a functional description of concepts is necessarily a representation in terms of a minimal set of observable functions. As opposed to constructs such as beliefs or intellectual theories about the mechanisms by which those functions are implemented, or in terms of function that can't yet be validated within observation.

In the conceptual space defined by the FMF the subjective relationships between concepts are defined by a given perspective. These relationships define the position of concepts in the conceptual space. In the segment of conceptual space shown in figure 7 there are a multitude of relationships connecting the fox to entities that define its various properties. For example, from the perspective of a comparison with a "mouse" the fox is "large". From the perspective of a comparison with a "horse" the fox is "small". In order to be able to retrieve all the relationships relevant to a given perspective, the represention of the conceptual space must be complete enough to store such perspectives.

\section{Constructs in Conceptual Space - Zoom In}

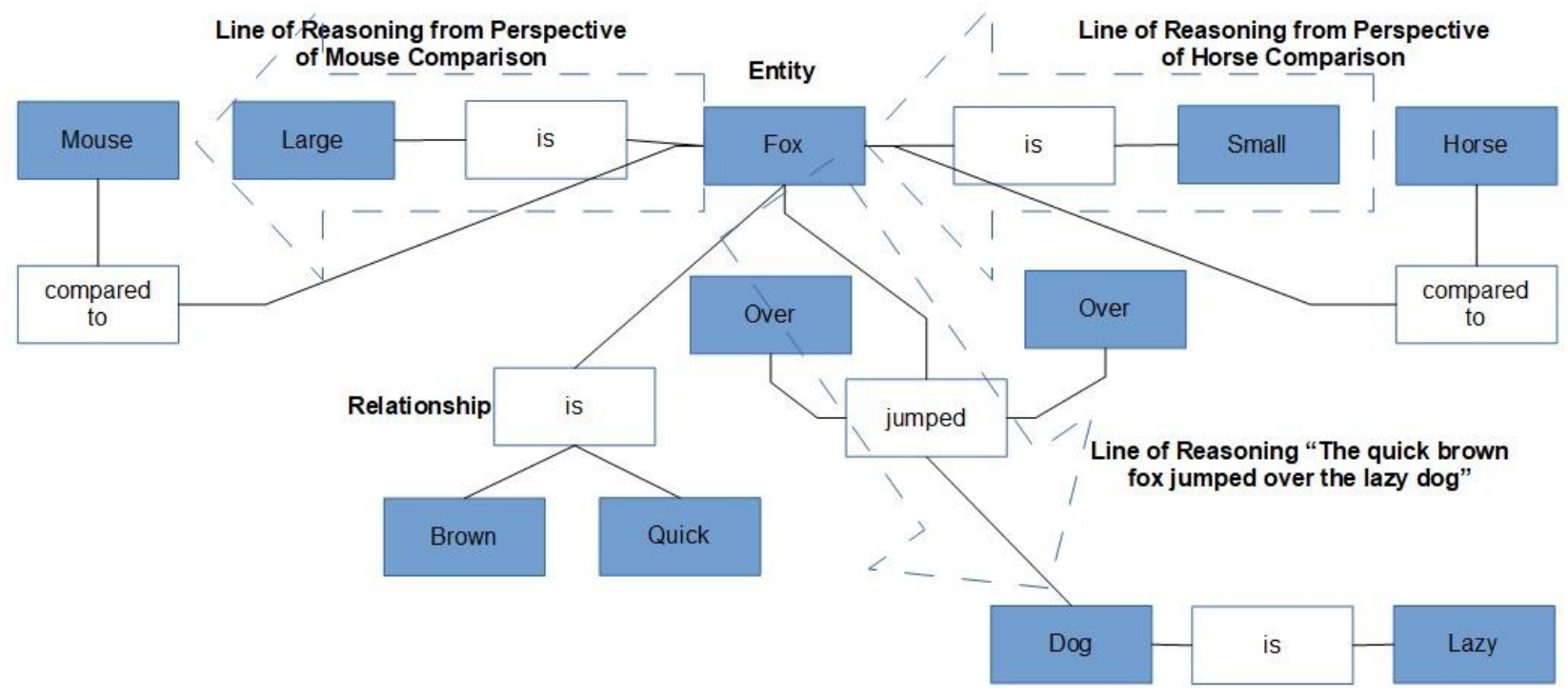

Figure 7: Depiction of relationships in conceptual space.

A collective conceptual space must also be defined for any group process to be driven by collective cognition. In the case of manufacturing, HCFM permits the creation of a common model of products and services in terms of what they can be used to do, and facilitates use of GCI in the design, manufacturing, sales, and other processes that can be used on those products or services. This collective cognition, and hence this modeling, is proposed to be required in order to have the capacity to reliably achieve certain classes of collective outcome, including reducing consumption to sustainable levels through massive decentralized cooperation not reliably achievable otherwise. HCFM is therefore essential to having the capacity to address the environmental degradation that has been linked to climate change [16]. 
Consciousness systems, and cognitive systems are both adaptive problem solving systems of computation, that is, they compute solutions to problems posed by their respective environments. From the point of view of the FMF, consciousness systems solve the problem of navigating awarenesses (the truth of any awareness received as input or directed as output). From the point of view of the FMF, cognitive systems solve the problem of navigating concepts (relationships that have been used to represent awarenesses). All adaptive problem solving systems that solve the problem of navigating between different truths through executing processes that compute how the system's awareness of one state (one truth) moves to awareness of another state (another truth) can be seen as systems of consciousness. And all adaptive problem solving systems that solve the problem of navigating between any representations of different entities through executing processes that compute how the system's representation of one entity (one state) moves to representation of another entity (another state) can be seen as systems of cognition. From this perspective awareness of the state of matter is a kind of distributed universal consciousness by which the distributed cognition of the universe computes the evolution of matter. Using this analogy to generalize the HCFM based models of consciousness and cognition so they apply to the basic physical principles by which matter evolves allows philosophical terms like "panpsychism" to be defined objectively, as well as having other implications in mathematics and physics described in this paper.

In any case, relationships defining the representation of an entity constitute the concept or "idea" of an entity. All truths can potentially be represented by such concepts, and all concepts are potentially generalizable. If convergence is generalizing categories representing static concepts or behavioral models, maximizing convergence is maximizing this ability to generalize, that is, maximizing the degree to which all concepts can be recategorized into common categories.

HCFM is a new formalization of a tradition that is very old. A large body of work has been done over thousands of years to define human-centric functional models of not only the human system, but of much of this entire existence that can be perceived. Since these traditions provide experientially verifiable definitions of terms that when defined intellectually are ambiguous, this in itself is a tremendous contribution to research in areas such as consciousness or cognition because they define the problems of consciousness and cognition experientially where no complete explanation of implementation exists. This experiential approach also potentially applies to all of existence, and when applied, in many cases it might permit the observer to more reliably achieve convergence of understanding. In other words, intellectual reasoning has a capacity to arrive at truth that is finite (limited to cases in which adequate reasoning and the facts to plug into that reasoning exist) and potentially unreliable (reliable only where such reasoning is simple enough to be accurately computed). Experiential reasoning has a capacity to arrive at truth that is infinite (the truth of an infinite number of observations can be experienced) and reliable (experience can reliably be observed wherever one has practiced awareness to the degree that an observation can be accurately identified as one's experience). The more experiential and less intellectual the discussion of cognition, potentially the more capable that discussion is of reliably converging on the truth. Yoga, Buddhism, and other eastern traditions are "existential" in that they aspire to be based on truths observed of this existence, rather than being based on any doctrine, though in their lowest form, like any other spiritual tradition, they may reduce to mere superstition. For this reason the "practice" of any doctrine has been suggested to be more important than the doctrine itself [21]. A sophisticated practitioner of any faith might have more in common with a sophisticated practitioner of any other faith because of the existential approach required for any understanding to become deeply self-consistent (convergent). An unsophisticated practitioner of any faith similarly might have more in common with the unsophisticated in any other faith in that convergence in their beliefs might be driven by superstition. 


\section{The Components of Human-Centric Conceptual Modeling}

The FMF defines the elements needed to implement HCFM in any discipline of study as those below:

$\begin{array}{ll}\begin{array}{l}\text { Component of Model } \\ \text { Functional modeling } \\ \text { (problems and solutions) }\end{array} & \begin{array}{l}\text { Description } \\ \text { Components are modeled only by function to remove prejudice for or } \\ \text { against any given implementation. }\end{array} \\ \text { Functional decomposition } & \begin{array}{l}\text { Functional components are decomposed into their most basic functional } \\ \text { building blocks for reuse. }\end{array} \\ \text { Functional domain bridging } & \begin{array}{l}\text { Different domains in which different functions are more fit in achieving } \\ \text { the same purpose are identified. These domains are bridged by using a set } \\ \text { of weights which identify the best function in each domain. } \\ \text { Every functional component is assigned some projected and actual fitness } \\ \text { in achieving its function. }\end{array} \\ \text { Functional fitness } & \begin{array}{l}\text { For functional components to persist they must display some degree of } \\ \text { stability in fitness to function. }\end{array} \\ \text { Functional stability } & \begin{array}{l}\text { For functional components to persist in a changing environment they must } \\ \text { have the ability to adapt their function. One of the most important } \\ \text { domains of adaptation is cooperation. Functional components must } \\ \text { interact according to the principles of decentralized cooperation in order } \\ \text { to maximize outcomes for each. Functions must be prioritized according } \\ \text { to the principles of centralized cooperation in order to maximize outcomes } \\ \text { globally. }\end{array}\end{array}$

Table 1: Components of a HCFM compliant model.

The criteria by which HCFM can be confirmed to have been applied are that all these functions can be observed to exist.

\section{Conclusions}

Systems (dynamic entities) respond to their environments through changing their states. These states reflect the truth of their awareness of their environment. HCFM has demonstrated the ability to converge a wide range of dynamic entities into the same model of behavior that spans all adaptive domains. Static entities have functions that reflect the truth of their outcomes but don't have behaviors through which the entity might change states in response to their environments. Static entities also have semantic relationships that determine whether they can be accommodated in the same conceptual space. HCFM has demonstrated the ability to converge all static entities into the same conceptual space in which all concepts are defined by function. HCFM also defines a minimal set of operations with which to increase capacity to navigate the conceptual space in which all of these static and dynamic entities are defined. In this sense HCFM alone has demonstrated the capacity to result in significant potential for convergence in concepts.

In addition, the ability to converge concepts is related to both the limits to cognitive capacity and this capacity for navigation. This paper has explored some of the limits to cognition. In the FMF the limits to cognitive capacity are defined by the size of the conceptual space, the resolution with which concepts can be located, which is related to the number of patterns in concepts that can be detected simultaneously, the ability to accurately navigate a number of such pattern detection (reasoning) processes consecutively, and other factors. The capacity for navigation is limited by whether the set of reasoning processes are minimal, which essentially equates to the simplicity of directions to the location of a concept (point in conceptual space), which defines whether complicated and lengthy paths can reliably be navigated. This paper has also explored the impact of HCFM on those cognitive limits, 
which is proposed to be to reduce them by making larger regions of the conceptual space accessible, and by increasing the ease and speed with which that increased conceptual space can be navigated.

This approach isn't limited to biological systems. From the perspective of this HCFM approach, all matter and energy might also be represented as dynamic problem solving systems that attempt to compute the influence of their environment through the evolution of their states. Current work is exploring how the HCFM perspective can potentially be used to define models of complex physical systems and complex mathematical relationships that reliably provide opportunities for greatly expanded and convergent insights in mathematics and physics. In particular, recent work by Wolfram has explored how simple relationships might be used many times in succession to define very complex systems such as the principles underlying the evolution of matter in the universe [18], and work by Penrose [19], [20] has explored how a kind of "awareness" of states might resolve fundamental inconsistencies in quantum mechanics and general relativity. Current work is exploring how these ideas might be combined with HCFM to systematically explore a much wider range of relationships that might be used to define those principles.

If cognition is an adaptive problem-solving system that solves the problem of adapting to achieve stability in its fitness to execute its functions (i.e. solving the problem of adapting to achieve cognitive well-being), then the purpose of improving cognition is to improve capacity to achieve well-being. This applies for both individuals and groups governed by systems of collective cognition. The purpose of this HCFM approach is to increase capacity to navigate all knowledge so that it is more reliably possible to navigate to cognitive well-being. Where intelligence (general problem solving ability) is modeled in the FMF as being the capacity to navigate a greater volume of conceptual space per unit time, the capacity to reliably navigate to cognitive well-being is represented in the FMF as cognitive enlightenment or wisdom. Intelligence is from this perspective is knowing how to design drift nets with the capacity to clear the entire sea of fish. Wisdom is knowing not to do so. If the minimal functional models of conceptual space that are obtainable through HCFM are more experiential than memory based (i.e. require awareness of experience rather than memory of an intellectual model that can't directly be validated through experience), then navigating knowledge through memory of relationships might be more closely related to intellect. While navigating knowledge through observation of function might be more closely related to wisdom. We would then expect intellect to limited by the capacity of memory. And we would expect wisdom to be limited by the capacity of observation. To be maximally useful, convergence would then be best served by utilizing observation driven HCFM to increase collective wisdom rather than simply gathering information in the collective memory to increase collective intelligence. Of course, this functional model also defines conscious well-being as existing in the consciousness system. And the importance of this distinction is understanding the difference between enlightenment in the consciousness and enlightenment in the cognition, which creates the possibility to become aware that well-being in the consciousness does not require cognition at all.

In addition to exploring why HCFM might be required to expand the limits on individual cognition, this paper has also explored why developing HCFM is required in order for a model of GCI to have a collective conceptual space to navigate, and why GCI might be required to expand the limits to group cognition. Since group cognition impacts the collective study of virtually every discipline, and since group cognition is required for collective convergence, HCFM is then critical to all research. Such a "theory of everything" might be uncomfortably ambitious for most.

A visionary is a painter of ideas big enough to reveal our fundamental challenge is not thinking outside the box, but realizing the box doesn't exist. There are those who see, those who can be shown, and those who cannot see. In order to become a great artist in the eyes of the world, a painter must find an 
audience who see from far enough away that the work in its entirety is revealed. Or they must find a critical mass of individuals who can be shown, those having the diligence to examine all the individual parts of a picture in isolation, so memory can piece the disconnected images together into a whole.

In convergence research, the concept of HCFM might be too big to be easily manageable within any research process. Funding convergence research is an exercise in identifying big ideas. However, policies of organizations typically must enforce ways of doing things that lead to predictable outcomes (like choosing how much risk to tolerate, and at what point is potential impact too speculative). By this definition, big, broad ideas that create the possibility of new outcomes are an exercise in disruption of the very policies tasked with identifying and funding convergence through such ideas. It may be true that entire classes of big, or broad ideas must then be systematically rejected by any policies even where those ideas might represent optimal research outcomes, simply because any policy can only accommodate repeated patterns. But if the lack of HCFM is a fundamental limit to individual and collective cognition as suggested in this paper, and therefore is a fundamental limit to existential challenges facing human civilization, the challenge of HCFM must be taken up regardless of its size.

\section{References}

[1] Oppl, S. Supporting the Collaborative Construction of a Shared Understanding About Work with a Guided Conceptual Modeling Technique. Group Decis Negot 26, 247-283 (2017). https://doi.org/10.1007/s10726-016-9485-7

[2] Robert Andrei Buchmann, Dimitris Karagiannis, Pattern-based Transformation of Diagrammatic Conceptual Models for Semantic Enrichment in the Web of Data, Procedia Computer Science, Volume 60, 2015, Pages 150-159, ISSN 1877-0509, https://doi.org/10.1016/j.procs.2015.08.114.

[3] S. Mohan, E. Choi and D. Min, "Conceptual Modeling of Enterprise Application System Using Social Networking and Web 2.0 "Social CRM System"," 2008 International Conference on Convergence and Hybrid Information Technology, Daejeon, 2008, pp. 237-244, doi:

10.1109/ICHIT.2008.263.

[4] J. Andrade, J. Ares, R. Garcia, J. Pazos, S. Rodriguez and A. Silva, "A methodological framework for viewpoint-oriented conceptual modeling," in IEEE Transactions on Software Engineering, vol. 30, no. 5, pp. 282-294, May 2004, doi: 10.1109/TSE.2004.1.

[5] Lukyanenko R., Parsons J. (2013) Is Traditional Conceptual Modeling Becoming Obsolete?. In: Ng W., Storey V.C., Trujillo J.C. (eds) Conceptual Modeling. ER 2013. Lecture Notes in Computer Science, vol 8217. Springer, Berlin, Heidelberg

[6] Lukyanenko R., Parsons J. (2013) Lightweight Conceptual Modeling for Crowdsourcing. In: Ng W., Storey V.C., Trujillo J.C. (eds) Conceptual Modeling. ER 2013. Lecture Notes in Computer Science, vol 8217. Springer, Berlin, Heidelberg

[7] Williams, Andy E. "A Human-centric Functional Modeling Framework for Defining and Comparing Models of Consciousness and Cognition.” PsyArXiv, 16 Apr. 2020. Web.

[8] Model for Human, Artificial \& Collective Consciousness (Part I), Journal of Consciousness

Exploration \& Research, Andy E. Williams, Vol 10, No 4 (2019), pages 250-269

[9] Model for Human, Andy E. Williams, Artificial \& Collective Consciousness (Part II), Journal of Consciousness Exploration \& Research, Vol 10, No 4 (2019), pages 270-293

[10] Williams, Andy E. “A Model for Artificial General Intelligence.” AfricArXiv, 20 Apr. 2020. Web. [11] Williams, Andy E. “A Model for General Collective Intelligence.” AfricArXiv, 30 Apr. 2020. Web. [12] Williams, Andy E. "Defining Functional Models of Artificial Intelligence Solutions to Create a Library That an Artificial General Intelligence Can Use to Increase General Problem Solving Ability." AfricArXiv, 27 Apr. 2020. Web.

[13] Williams, Andy E. "The Need for General Collective Intelligence in Addressing the Sustainable Development Goals", https://www.researchsquare.com/article/rs-18022/v1 
[14] Williams, Andy E. “The Case for General Collective Intelligence Rather Than Artificial General Intelligence Being the Most Important Human Innovation in the History and Future of Mankind." AfricArXiv, 20 Apr. 2020. Web.

[15] Williams, Andy E. "A Mathematical Model for Identifying Truth in Observations Made Within Individual Human Self-awareness.” AfricArXiv, 27 Apr. 2020. Web.

[16] GCI and Human-Centric Design

[17] Kahneman, Daniel. Thinking, Fast And Slow. New York : Farrar, Straus And Giroux, 2011.

[18] Stephen Wolfram, A Class of Models with the Potential to Represent Fundamental Physics, accessed from https://arxiv.org/abs/2004.08210

[19] Penrose, R. 1987. Quantum physics and conscious thought. In B. Hiley and Peat, eds., Quantum Implications: Essays in Honor of David Bohm. New York: Methuen.

[20] A. Ekert, R. Jozsa, R. Penrose and Roger Penrose 1998Quantum computation, entanglement and state reductionPhil. Trans. R. Soc. A.3561927-1939

http://doi.org/10.1098/rsta.1998.0256

[21] Smith, Huston. The World's Religions: Our Great Wisdom Traditions. [San Francisco]: Harper San Francisco, 1991. Print 